\title{
Modified interferon $\gamma$ targets key cells responsible for liver fibrogenesis
}

The cytokine interferon (IFN)- $\gamma$ has potent antifibrogenic properties in vitro. However, this molecule has proven disappointing in clinical trials; one of the reasons for this is that off-target effects are common owing to the presence of IFN- $\gamma$ receptors on nearly all cell types. Ruchi Bansal and colleagues have investigated a method to target IFN- $\gamma$ to hepatic stellate cells (HSCs), which are the key cells responsible for liver fibrogenesis.

"In the past, our group has developed a cyclic peptide that has specific affinity for platelet-derived growth factor beta receptor (PDGF $\beta R$ )," explains Bansal. “To target IFN- $\gamma$ to HSCs, we used our PDGF $\beta$ R recognizing peptide ... as $\mathrm{PDGF} \beta \mathrm{R}$ is highly over-expressed in activated HSCs in liver fibrosis." The group conjugated IFN- $\gamma$ to this cyclic peptide and analyzed the biological activity and the PDGF $\beta R$ binding specificity of these constructs using in vitro and in vivo models.
The modified IFN- $\gamma$ constructs were found to retain their biological activity and showed PDGF $\beta$ R-specific binding to fibroblasts and HSCs in vitro. The in vivo model demonstrated that modified IFN- $\gamma$ inhibited HSC activation and attenuated liver fibrosis. Furthermore, adverse affects were reduced or absent compared with unmodified IFN- $\gamma$.

"Further studies will be undertaken to examine the therapeutic and adverse effects of these constructs in other animal models of liver fibrosis," concludes Bansal. "Parallel to these proof-of-concept studies, a preclinical research program has been set up in coordination with experts in the field to prepare for clinical trials."

Isobel Franks

Original article Bansal, R. et al. A novel engineered targeted interferon gamma blocks hepatic fibrogenesis in mice. Hepatology doi:10.1002/hep.24395 